\title{
Shaping the zebrafish myotome by differential friction and active stress
}

\author{
S. Tlili ${ }^{2},{ }^{1}$ J. Yin $\#,{ }^{1}$ J.-F. Rupprecht,${ }^{1}$ G. Weissbart,${ }^{1}$ J. Prost,${ }^{1,2}$ and T. E. Saunders ${ }^{* 1,3,4}$ \\ ${ }^{1}$ Mechanobiology Institute, National University of Singapore, 5A Engineering Drive 1, 117411, Singapore. \\ ${ }^{2}$ Laboratoire Physico Chimie Curie, Institut Curie, \\ PSL Research University, CNRS UMR168, 75005 Paris, France. \\ ${ }^{3}$ Department of Biological Sciences, National University of Singapore, Singapore. \\ ${ }^{4}$ Institute of Molecular and Cell Biology, Agency for Science, \\ Technology and Research (A*Star), Biopolis 138673, Singapore. \\ \# denotes equal contribution. \\ * for correspondence: dbsste@nus.edu.sg
}

(Dated: December 22, 2018)

\begin{abstract}
Organ formation is an inherently biophysical process, requiring large-scale tissue deformations. Yet, understanding how complex organ shape emerges during development remains a major challenge. During fish embryogenesis, large muscle segments, called myotomes, acquire a characteristic chevron morphology, which is believed to play a role in swimming. The final myotome shape can be altered by perturbing muscle cell differentiation or by altering the interaction between myotomes and surrounding tissues during morphogenesis. To disentangle the mechanisms contributing to shape formation of the myotome, we combine single-cell resolution live imaging with quantitative image analysis and theoretical modeling. We find that, soon after its segmentation from the presomitic mesoderm, the future myotome spreads across the underlying tissues. The mechanical coupling between the myotome and the surrounding tissues is spatially varying, resulting in spatially heterogeneous friction. Using a vertex model, we show that the interplay of differential spreading and friction is sufficient to drive the initial phase of myotome shape formation. However, we find that active stresses, generated during muscle cell differentiation, are necessary to reach the acute angle of the myotome observed in wildtype embryos. A final ingredient for formation and maintenance of the chevron shape is tissue plasticity, which is mediated by orientated cellular rearrangements. Our work sheds a new light on how a spatio-temporal sequence of local cellular events can have a non-local and irreversible mechanical impact at the tissue scale, leading to robust organ shaping.
\end{abstract}

12 The formation of complex organ shape requires the in13 tegration of genetic information 1,4 with mechanical 14 processes such as directed cell division and rearrange15 ments [5] 11] and interactions between tissues [12]. The 16 highly robust form of organs [7] suggests that forming a 17 precise shape is essential. However, it remains an open 18 question how different biophysical and genetic processes 19 dynamically interact during organogenesis [13.

20 In the zebrafish embryo, precursors of myotomes, 21 somites, start to bend into a chevron shape soon af22 ter segmentation [14]. Posterior trunk and tail somites 23 emerge from the presomitic mesoderm (PSM), Fig. 1a., 24 whilst anterior counterparts are generated from the meso25 derm during gastrulation. Somites are specified by pe26 riodic segmentation around every $30 \mathrm{~min}$ [14 16] and ${ }_{27}$ they give rise to slow and fast-twitch muscle fibres, der28 momyotome and various types of progenitor cells [17 21]. 29 The developmental stage of a specific somite is denoted 30 by $\mathrm{SN}$, where $\mathrm{N}$ counts the number of already formed 31 somites, with a newly specified somite denoted as stage ${ }_{32} \mathrm{~S} 1$, Fig. 1a. The final myotome consists of slow muscle 33 fibres, whose progenitors are initially located close to the 34 notochord, and multinucleated fast fibres, whose progen35 itors are initially located more laterally, Fig. $1 \mathbf{b .}$

36 The mature myotome has a distinctive V ("chevron") ${ }_{37}$ shape, 14, Fig. 1a, which is thought to be important 38 for swimming [22]. A number of hypotheses have been 39 proposed to explain chevron formation, including roles 40 for: the swimming motion itself [23]; older myotome seg-
41 ments acting as templates for younger segments [24, 25]; 42 tissue shear flow between the notochord and the develop43 ing myotome [26; the interplay between intra-segmental 44 tension and fixed myotome boundaries 14 .

45 Here, we combine quantitative analysis of in vivo imag46 ing data with modeling to show that a robust chevron 47 shape emerges from the interplay between short-ranged 48 processes (including cell differentiation and cell neigh49 bour exchanges) and long-ranged mechanical processes 50 mediated by the coupling between developing somites 51 and their surrounding tissues.

${ }_{52}$ Symmetry breaking in the somite occurs early af${ }_{53}$ ter segmentation from the PSM: We imaged somites 54 at subcellular resolution inside the developing embryo, 55 from their segmentation within the PSM (earliest S-2) to 56 mature myotome stage (S5 onward), Fig. 1 c-d, Meth57 ods, Supplementary Movie 1. Immediately after seg58 mentation, somites are approximately cuboidal 27, 28, ${ }_{59}$ Fig. 1d-e and Supplementary Movie 2. Quantifying the 60 somite contours over $8 \mathrm{~h}$, we observe that the process of ${ }_{61}$ chevron formation occurs during phases S1 to S5, Fig. 1e 62 and Supplementary Fig. 1. Somite volume is approxi63 mately constant during the $7 \mathrm{~h}$ following segmentation 64 (Supplementary Fig. 2c). Immediately after segmenta65 tion the somites begin to change shape, with flattening 66 in the medial-lateral (ML) axis, leading to an increased 67 contact area with the medially underlying tissues (noto${ }_{68}$ chord, neural tube and ventral tissues), Fig. 11e-f, Sup- 
${ }_{69}$ plementary Movie 2 and Supplementary Fig. 1.

70 Concurrently with spreading, we observe symmetry71 breaking in the somite shape along the anterior-posterior 72 (AP), Fig. 1e. In the medial region a "U" shape emerges 73 that always points toward the anterior of the embryo. 74 This "U" subsequently sharpens into the chevron shape, 75 Fig. 1 e.

Chevron angle is impacted by both internal and 77 external factors to the myotome: The shape of the 78 myotome is known to be sensitive to a range of per-
79 turbations 29,30 , including to: (i) signaling pathways 80 31, 32; (ii) the surrounding extracellular matrix (ECM) 81 [33, 34]; and (iii) the surrounding tissues [35, 36]. Un${ }_{82}$ der perturbation, the myotome becomes more "U"-like or 8 even flat. We are unaware of perturbations that sharpen 84 the chevron, suggesting that the shape is tightly con85 trolled and may be evolutionarily optimised. We quan${ }_{86}$ tified the chevron angle in a range of different condi87 tions, using genetic ( $s m o^{-/-}$, Supplementary Movie 3) 88 and drug perturbations (Cyclopamine, Shh pathway in89 hibitor, Supplementary Movie 4). We complemented this 90 with data from the literature, Fig. $1 \mathrm{~g}$ and Methods Table 91 1. The chevron angle increases linearly from $90^{\circ}$ towards ${ }_{92} 180^{\circ}$ with decreasing slow muscle number. In contrast, 93 altering of the extracellular matrix at the interfaces of 94 somites and axial tissues (e.g. through Col15a $1 \mathrm{a}^{M O}$, ${ }_{95}$ Fukutin $^{M O}$ or lamc1-/- , see Methods Table 1) dras96 tically reduces the chevron angle while the number re97 mains largely unchanged, Fig. 1g. These results suggest 98 that both muscle cell differentiation (intrinsic to each 99 somite) and ECM interactions (at the interface between 100 somites and surrounding tissues) are critical in forming 101 the chevron.

102 Somite deformation occurs prior to fast mus${ }_{103}$ cle fibre elongation: Concurrent with the tissue shape 104 changes, cells within the somite begin differentiation 162 105 into specific muscle fibres [17, 20, 21, 27, 37, 38. The 106 most-medial layer of cells undergoes differentiation into 164 107 slow muscle fibres at the onset of somite segmentation, 165 ${ }_{108}$ Fig. 1b [21]. Slow muscle fibres, which are epithelial109 like before segmentation, rapidly elongate along the AP110 axis until they span the somite compartment. To quan111 tify the dynamics of slow muscle elongation, we used a ${ }_{112}$ Fourier transform method to analyse the evolution in cel${ }_{113}$ lular anisotropy within the somite, Fig. 1 $1 \mathbf{h}-\mathbf{i}$, Methods ${ }_{114} 39$. We find that signatures of future slow muscle fibre 115 elongation are apparent even before segmentation, and 116 that these cells rapidly extend over the next $100 \mathrm{~min}$, ${ }_{117}$ Fig. 1j. In contrast, fast fibres elongation occurs sig118 nificantly later, at around $250 \mathrm{~min}$, Fig. 1]. Comparing 17 119 with Fig. 1e, we see that the chevron is apparent around $120200 \mathrm{~min}$ after segmentation, yet fast fibres only fully elon-
${ }_{121}$ gate around this time. Despite fast muscle fibres repre122 senting $>80 \%$ of somitic cells, the future myotome ac${ }_{123}$ quires the characteristic chevron shape before most of 124 these cells have begun to elongate.

${ }_{125}$ Spatio-temporal variation in somite-tissue cou126 pling correlates with the chevron shape: As per-
${ }_{127}$ turbations to surrounding tissues and ECM alter the my128 otome shape, we explored the mechanical coupling be129 tween somites and surrounding tissues. We used 2D op130 tic flow to quantify the cellular velocity fields inside the 131 somites (at different medial-lateral locations) and in the 132 adjacent notochord and neural tube, Fig. 2a-b, Methods. ${ }_{133}$ We computed the averaged in-plane 2D velocity fields in 134 the medial-lateral (ML) axis, Supplementary Fig. 3, as 135 the shear velocities along the ML axis were comparatively 136 small.

To gain insight into the physical coupling between tis138 sues, we focused on relative tissue velocities. We primar139 ily considered the velocity component along the AP-axis ${ }_{140}$ for each tissue ( $V_{\text {not }}$ (notochord), $V_{\text {som }}$ (somite) and $V_{\mathrm{NT}}$ ${ }_{141}$ (neural tube)), Methods. We define the shear velocity ${ }_{142}$ within the somite $V_{\text {chev }}$ as the relative difference in the 143 velocity of cells at the DV-midline and of those in more 144 dorsal positions, Fig. 2b, along with similar shear ve145 locities between somites and surrounding tissues: $V_{\mathrm{som}}^{\mathrm{NT}}$ ${ }_{146}$ (relative somite velocity compared to neural tube); and ${ }_{147} V_{\text {som }}^{\text {not }}$ (relative somite velocity compared to notochord). ${ }_{48}$ Lastly, we define a shear velocity $V_{\text {not }}^{\mathrm{NT}}$ between the no149 tochord and neural tube, Fig. $2 \mathrm{~b}$.

Each of these shear velocities has distinct behaviour, ${ }_{151}$ Fig. 2c, kymographs Fig. 22d-d" and Supplementary 152 Fig. 4. In agreement with the chevron formation 153 timescale identified in Fig. 1f, $V_{\text {chev }}<0$ during the first ${ }_{154} 5 \mathrm{~h}$ after segmentation, Fig. 2d'. Within this time, the 155 notochord moves more posteriorly than the neural tube ${ }_{156}$ during chevron formation, as $V_{\text {not }}^{\mathrm{NT}}$ remains positive after 157 segmentation, Fig. 2c. Hence, somites are not passively 158 deformed by an underlying tissue shear, in which case the 159 chevron would point toward the embryo posterior. Soon 160 after segmentation $V_{\mathrm{som}}^{\mathrm{NT}} \approx 0$, implying that the somite 161 and neural tube move concomitantly, Fig. 2c,d. Simi162 larly, before segmentation, future somites and notochord 163 move concomitantly, Fig. 2 d". In contrast, $V_{\text {som }}^{\text {not }}<0$ 164 throughout the $6 \mathrm{~h}$ after segmentation, Fig. 2c,d", im165 plying that somites move in the anterior direction relative 166 to the notochord.

${ }_{167}$ We complemented this analysis with live imaging of 168 embryos injected with lyn-Kaede, Methods. By switching 169 the Kaede at a somite boundary, we observed the differ170 ential movement of the notochord and neural tube with 171 respect to the somite, Fig. 2 g, consistent with the above 172 velocity maps. From these observations, our hypotheses 173 are: (i) that somites and neural tube are weakly mechan174 ically coupled prior to segmentation but strongly linked 175 after segmentation; (ii) that somites and notochord are 176 mechanically coupled prior to segmentation and uncou177 pled afterwards.

${ }_{178}$ Mechanical coupling between tissues varies in 179 time: To explore whether temporal changes in the rel180 ative movements between tissues are correlated with 181 changes in physical interactions between these tissues, we ${ }_{182}$ examined the distribution of actin and the ECM compo183 nent laminin, 40, Fig. 2e-f and Supplementary Movie 51846 . Before segmentation, future somitic cells are in contact 
185 with the notochord, Fig. 2f; while after segmentation, a 240 We model this compartmentalisation by increasing the 186 gap between these cells and the notochord emerges, to- 241 tension $\gamma$ along the somite/somite boundaries, Fig. 33b, 187 gether with the appearance of large actin fibres. Such ${ }_{242}$ Methods. Lastly, to simulate growth and division within 188 loss of contact suggests a reduced friction between the ${ }_{243}$ the PSM and tailbud, we continuously add new cells at 189 notochord and the slow muscle fibres.

190 In contrast, cells in the PSM appear in contact with 191 the neural tube, with progressive actin accumulation be192 tween the tissues, Fig. 22p. After segmentation, a layer of ${ }_{193}$ laminin appears between the somitic cells and the neu${ }_{194}$ ral tube, Fig. 2 e', which suggests that mechanical cou${ }_{195}$ pling between the neural tube and somitic cells further 196 increases. Other molecules could also contribute to ad197 hesion, e.g. integrin and fibronectin, whose localisation 198 are tightly regulated during somite formation [41.

199 Following [42, 43, we expect strongly (weakly) ad200 hered tissues to have a high (low) effective interfacial ${ }_{201}$ friction coefficient. Such a framework has proved fruitful 202 in understanding tissue-tissue interactions during early 203 zebrafish morphogenesis [44. Below, we incorporate this 204 idea - along with somite spreading and cell differentiation 205 - within a vertex model to test how tissue-tissue coupling 206 drives the chevron shape of the myotome.

${ }_{207}$ Simulating tissue shape formation within a vertex ${ }_{208}$ model: The chevron first emerges on the medial side of 209 the somite, which includes slow muscle fibres and the 210 most-medial future fast fibres. We simulate an average ${ }_{211} 2$ D layer of cells located within this medial compartment ${ }_{212}$ of each somite, Fig 3 a. We do not distinguish specific 213 muscle types.

${ }_{214}$ Each cell is described by a polygon whose summits, 215 called vertices and denoted by $X_{i}$, correspond to the 216 edges of cell-cell interfaces. Cellular movements and de${ }_{217}$ formations are described through the dynamics of the ${ }_{218}$ cell vertices, which is set by the following force-balance 219 equation:

$$
\nu_{i} \dot{X}_{i}=\nabla_{X_{i}} E+F_{\text {elongation }}
$$

222 This equation has three critical elements. (i) spatially${ }_{223}$ dependent friction: $\nu_{i}$ represents the friction on vertex $i$ 224 exerted by the underlying tissues, (ii) active stress forces, 225 denoted $F_{\text {elongation }}$ which are generated by the elongation ${ }_{226}$ of slow muscle fibres, Methods, and (iii) cell-scale forces ${ }_{227}$ regulating cell shape, $\nabla_{X_{i}} E$. Following [45, we consider

$$
{ }_{228}^{228} E=\sum_{C, I}\left[\lambda\left(A_{C}-A_{0}\right)^{2}+\beta\left(P_{C}-P_{0}\right)^{2}+\gamma_{I} L_{I}\right],
$$

230 where $A_{0}\left(P_{0}\right)$ represents the preferred area (perimeter) ${ }_{231}$ of a cell $C, A_{C}\left(P_{C}\right)$ the actual area (perimeter) of a cell 232 at a given time, and $L_{I}$ the length of cell-cell interface ${ }_{233} I$, Methods. $\lambda$ represents the pressure forces involved ${ }_{234}$ in cell area regulation, while $\beta$ and $\gamma_{I}$ represent the 235 strength of cell- and interface-dependent tensions respec236 tively. Following [46], we introduce stress fluctuations ${ }_{237}$ through stochastic modulation in the tension of each ${ }^{238}$ cell-cell contact, Methods. After segmentation, no cel239 lular exchanges with neighbouring somites are observed. 244 the posterior end of the tissue, at a rate determined by 245 the segmentation clock [16].

${ }_{246}$ Somite spreading and differential friction are suf247 ficient to generate a shallow chevron shape: We 248 first tested in the model the effects of spatially vary249 ing friction and somite spreading. To simulate the wave 250 of spreading we varied the target area of each cell $A_{0}$ : ${ }_{251} A_{0}(t, x)=A_{0}+\left(A_{f}-A_{0}\right) \exp \left(-\left(t-\tau_{\text {seg }}^{N}\right) / \tau_{\text {spread }}\right)$ with ${ }_{252} \tau_{\text {spread }}=200 \mathrm{~min}$ extracted from experiment, Fig. 3r-c', ${ }_{253}$ and $\tau_{\text {seg }}^{N}$ is the segmentation date of the $N$-th somite. ${ }_{254}$ During spreading, each cell has a constantly increasing 255 target area and hence exerts pushing forces on its neigh256 bouring cells. We first considered somite spreading with 257 uniform friction, Fig. 3c". Along the DV axis of the 258 somite, all cells have the same target area and spread to259 gether. However, along the AP axis the cells are not at 260 the same stage of spreading. Newer (and subsequently ${ }_{261}$ smaller) somites have a higher spreading rate than more 262 anterior (older) somites, resulting in a net force along the ${ }_{263}$ central part of more anterior somites and a slight bend264 ing towards the head occurs. However this bending is 265 insufficient to irreversibly deform the somites; they relax 266 once spreading is finished, Supplementary Movie 7.

${ }_{267}$ We next introduced spatially inhomogeneous friction 268 within the model, Fig. 3 $3 \mathrm{~d}-\mathbf{d}$ '; $\nu_{i}$ depends on vertex po269 sition $X_{i}$. After segmentation, we increase the friction 270 coefficient over the neural tube and ventral tissues while 271 decreasing the friction coefficient over the notochord, ${ }_{272}$ Fig. 3d'. Combining spreading with non-uniform fric273 tion gives rise to clear symmetry breaking, with somites 274 deforming into a shallow chevron, Fig. 3d" and Supple275 mentary Movie 8. As cells lying above the notochord slide 276 faster than those located more dorsally, the stress asso277 ciated to the somite spreading creates a DV-shear that ${ }^{278}$ deforms somites into a U shape. Such tissue deformation 279 also alters individual cell shapes. If the tissue is suffi280 ciently plastic (i.e. frequent cell rearrangements), then ${ }_{281}$ cell rearrangements relieve stress induced by the shape 282 changes, resulting in a sharpening of the somite bound283 ary and the emergence of a stable but shallow chevron in ${ }_{284}$ early somites. However, this shape does not propagate to 285 younger somites as tissue spreading is insufficiently rapid 286 to trigger cell rearrangements in later segments. There${ }_{287}$ fore, incorporating realistic parameters (derived where ${ }_{288}$ possible from experiments) within such a model cannot ${ }_{289}$ generate a sharp chevron similar to wildtype embryos.

290 Active stress due to muscle fibre differentiation 291 modulates chevron angle: Slow muscle fibres start to 292 elongate soon after somite segmentation from the PSM, ${ }_{293}$ Fig. [1j. Such elongation likely exerts a shear stress on 294 the more lateral layers of future fast muscle fibres. To 295 model the mechanical constraints imposed by the layer 296 of slow muscle fibre, we used active gel theory, which 
297 is a hydrodynamic description of the acto-myosin cortex 298 that encompasses contractility and filament polymerisa299 tion through a local active stress tensor [47, Methods. $300 \mathrm{We}$ considered a traceless active stress to discriminate its 301 contribution from somite spreading. The positive compo302 nent (extension) of the active stress is orientated along 303 the AP-axis, in line with muscle fibre elongation, with 304 a corresponding negative component (contraction) orien305 tated along the DV-axis, Fig. 3e. We assume that the 306 active stress is maximal at the start of slow muscle elon307 gation, with a further linear decrease to zero by the end 308 of slow muscle elongation, Fig. 3e', which leads to a con309 vergence (DV-axis)-extension (AP-axis) wave within the 310 somites. The inclusion of such orientated active stress is 311 then sufficient to shape the tissue into a stable and sharp 312 chevron shape, Fig. 3e" and Supplementary Movie 9.

${ }_{313}$ Model predictions for myotome shape under per314 turbations: As shown in Fig. 1g, the chevron shape 315 changes with slow muscle number. Within our model, 316 this corresponds to changing the active stress, but leav317 ing other components unchanged. Without active stress, 318 the model predicts a transient shape deformation in the 319 somite before relaxing. These dynamics are strikingly 320 similar to $\mathrm{smo}^{-/-}$embryos, where there is no slow muscle 321 specification, Supplementary Movie 3. Intermediate lev322 els of active stress in the model result in reduced chevron 323 angle, as observed experimentally, Fig. 3f. Perturbations 324 to the interactions between tissues (e.g. Col15a1a $\left.{ }^{M O}\right)$ 325 likely change tissue-tissue coupling, thereby reducing the 326 effects of differential friction. Reducing friction hetero327 geneity within the model (while keeping active stress) ${ }_{328}$ results in a mild but stable bending of the myotome, 329 consistent with experiments, Fig. 3f and Supplementary 330 Movie 10.

${ }_{331}$ Dynamics of chevron formation: We next challenge 332 the model capacity to reproduce the tissue dynamics ob${ }_{333}$ served during chevron formation. In both experiments 334 and simulations, we quantified the tissue velocity, Fig. 4a 335 anisotropic strain rate (ASR), Fig. $4 \mathbf{b}$ and isotropic strain 336 rate, Supplementary Fig. 6. By definition, the ASR pro${ }_{337}$ vides the local tissue expansion direction. Common fea338 tures between the ASR fields in experiments and simu339 lations (Fig. 4 $\mathbf{a}^{\prime}, \mathbf{b}^{\prime}$ ) are: (i) in S1, somites undergo DV340 convergence and AP-extension (purple bars, Fig. $4 \mathbf{b}$ ), 341 correlating with the onset of slow muscle elongation, ${ }_{342}$ Fig. $1 \mathbf{j}$; (ii) in $\mathrm{S} 2$ somites, the ASR is near zero, (iii) ${ }_{343}$ from S3 to S6, somites undergo shear between central 344 and lateral regions.

345 Our interpretation of the ASR field is that active elon${ }_{346}$ gation of slow muscle cells is maximal at somite S1, gen${ }_{347}$ erating an extensile stress along the AP-axis that com${ }_{348}$ presses both the PSM and anterior somites. Such com349 pression pattern is similar to the one produced by a single 350 cell actively extending in a passive tissue, Supplementary ${ }_{351}$ Fig. 5, yet at the larger tissue level. To test this interpre352 tation, we laser ablated a region of newly formed somitic 353 tissue, Fig. 4c', Supplementary Movie 11 and Methods.
354 We observed a rapid relaxation of neighbouring tissues 355 towards the ablated tissue, confirming that newly seg356 mented somites are pushing their neighbours.

357 We then investigated the role of tissue plasticity. In a 358 purely elastic material, the somite shape would eventu359 ally relax, since the shear stresses generated by cell elon360 gation and spreading are transient. A plastic/viscous361 like behaviour is therefore required to acquire a stable 362 chevron shape. Within the vertex model, we imple363 mented passive cell rearrangements (Fig. $4 \mathrm{~d}$ ). Due to 364 the shear forces emerging in the model, passive cellu365 lar rearrangements are naturally oriented along the ASR 366 (Fig. 4f) indicating that the bulk somitic tissue has a 367 plastic-like behaviour [48.

368 Experimentally, we observe that tissue flows do not 369 generate large cell deformations, Supplementary Fig. 8, 370 which suggests the existence of cell rearrangements [49, 371 50]. Cell divisions can also relax cell shape [49]; however, 372 we found only infrequent cell divisions during myotome 373 formation, with less than $10 \%$ of cells dividing during 374 the whole process.

We explicitly show how cell rearrangements occur by 376 tracking cellular shapes in 3D inside S1, S2 and S3 377 somites using high temporal resolution movies, Fig. 4 d' 378 and Supplementary Movie 12. To correlate them with 379 the ASR, we superimposed the rearrangements in time 380 over an ASR field map, Fig. 4e,e' and Methods. Cellular 381 rearrangements are indeed closely aligned with the ASR, ${ }_{382}$ Fig. 4f, in agreement with our theoretical predictions.

${ }_{383}$ While intra-somite cell rearrangements are needed, 384 inter-somite cell exchanges ought to be prevented to 385 preserve the somite shape. Based on our simula386 tions, we expect somite-somite interfaces to be rough in $387 t b x 6^{-/-}$embryos, in which somites compartmentalisa388 tion is abolished. By using lyn-Kaede to define bound389 aries within $t b x 6^{-/-}$embryos, we indeed see greater 390 inter-compartmental mixing, Supplementary Fig. 7b-c. ${ }^{391}$ We note that it has been previously shown that using a 392 heat-shock inducible Tbx6, somite shape can be rescued 393 in $t b x 6^{-/-}$embryos, showing that the chevron forma394 tion is an emergent property, i.e. that it is not due to a 395 template mechanism [51].

${ }_{396}$ Conclusion: During myotome formation, somites are 397 under mechanical stress from both internal (somite 398 spreading, cell elongation) and external (tissue-tissue 399 coupling) processes. Combining our experimental and 400 cell-based numerical approaches, we propose the follow${ }_{401}$ ing sequence of mechanical events leading to chevron402 shaped myotomes: (i) increased line tension between de403 veloping somites leads to mechanically segmented cell 404 compartments, Fig. 3b; (ii) somite differential spreading ${ }_{405}$ (Fig. 3c) leads to a pressure gradient along the AP axis, 406 which, combined with the onset of a differential friction 407 along the DV axis (Fig. 3d), leads to a buckling instabil408 ity; and (iii) muscle fibre elongation further contributes 409 to buckling (Fig. 3e), which trigger cell rearrangements 410 that maintain a stable chevron shape. Our 2D model in${ }_{411}$ corporates features resulting from the $3 \mathrm{D}$ dynamics of the 
${ }_{412}$ somite, yet neglects cell heterogeneities within the fast- 436 in the presence of differential tissue-tissue frictions [56. ${ }_{413}$ muscle cell population, Supplementary Fig. 8. Though ${ }_{437}$ Therefore, our work suggests that both tissue-tissue in${ }_{414}$ we cannot discount other possible mechanisms, our model 438 teractions and tissue positioning can play a key role in 415 is minimal yet sufficient to recapitulate the dynamics of 439 shaping organs.

${ }_{416}$ somite shape formation in both wildtype and perturbed ${ }_{440}$ Acknowledgements We thank Philip Ingham for sup417 embryos.

${ }_{418}$ Recent works have shown (i) how active stress can 419 generate complex flows within in vitro tissues [53/55], ${ }_{420}$ (ii) how tissue-tissue friction affects tissue flows during ${ }_{421}$ early zebrafish embryogenesis [44] and (iii) how rheolog${ }_{422}$ ical properties set the shape of the zebrafish PSM and ${ }_{423}$ tailbud [46. Here, we integrate these approaches in the 424 vertex-model framework to understand the shaping of a 425 functional organ in terms of the interplay between (i) ac426 tive stresses generated by muscle cell differentiation, (ii) ${ }_{427}$ spatially heterogeneous friction and (iii) tissue plasticity. ${ }_{441}$ port with zebrafish experiments. We acknowledge fund${ }_{442}$ ing from a Singapore National Research Foundation ${ }_{443}$ Fellowship awarded to T.E.S. (grant No. 2012NRF444 NRFF001-094), an HFSP Young Investigator Grant 445 awarded to T.E.S. (grant no. RGY0083/2016), and the ${ }_{446}$ Mechanobiology Institute. Fish facilities were provided 447 by the Institute of Molecular and Cellular Biology, Sin${ }_{448}$ gapore. We thank MBI Science Communications for as449 sistance with graphics as well as Olivier Hamant and ${ }_{450}$ François Graner for critical reading of the manuscript.

${ }_{451}$ Author contribution J.Y. and T.E.S. planned the ex${ }_{452}$ periments, S.T. and T.E.S. planned the image analysis, ${ }_{428}$ It is interesting to compare with somite formation in 453 and S.T., J.-F.R., J.P. and T.E.S. planned the theoreti${ }_{429}$ other vertebrate systems, such as the chicken and mouse ${ }_{454}$ cal analysis. J.Y. performed all experiments except laser ${ }_{430}$ embryos whereby somites do not acquire a chevron shape. 455 ablation (by S.T.). S.T. performed the image analysis. ${ }_{431}$ While in our model the notochord needs to be centred to ${ }_{456} \mathrm{~S}$. T. and J.-F.R. developed the vertex model with in${ }_{432}$ generate the chevron shape, in mouse and chick embryos ${ }_{457}$ puts from G. W. and J.P., particularly regarding mod${ }_{433}$ the notochord is off-centred and located towards the ven- 458 elling of active stress. S.T., J.-F.R. and T.E.S. wrote the ${ }_{434}$ tral border (see Supplementary Fig. 9). Given such tissue 459 manuscript, with all authors contributing to manuscript 435 arrangement, we do not expect somites to buckle, even 460 preparation. We have no competing interests.

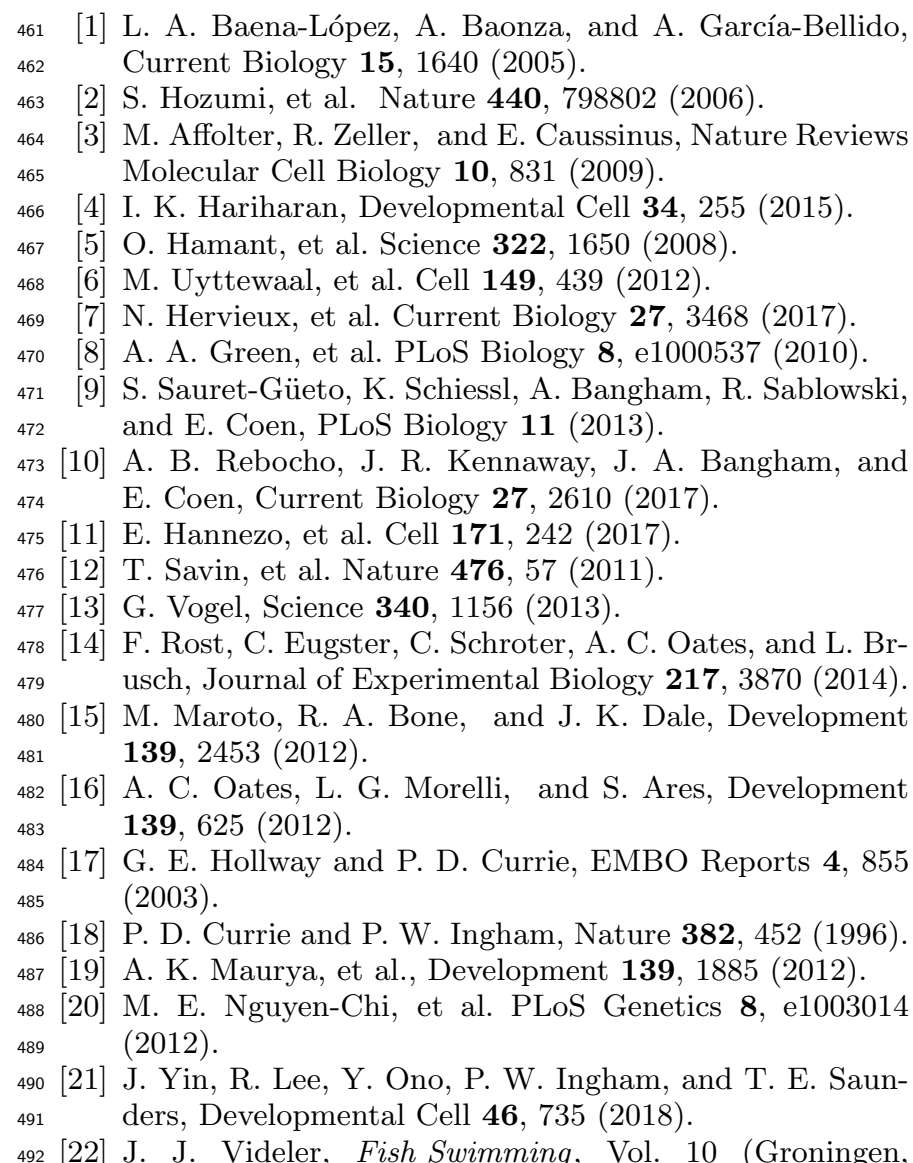

$493 \quad$ Springer, 1993).

494 [23] W. van Raamsdonk, W. Mos, G. Tekronnie, C. W.

495 Pool, and P. Mijzen, Acta morphologica NeerlandoScandinavica 17, 259 (1979)

497 [24] W. van Raamsdonk, et al. Zeitschrift für Anatomie und

$498 \quad$ Entwicklungsgeschichte 145, 321342 (1974).

499 [25] S. E. Windner, et al. Development 142, 1159 (2015).

500 [26] J. S. Turner, The Tinker's Accomplice (Cambridge, Har-

$501 \quad$ vard University Press, 2010).

502 [27] H. L. Stickney, M. J. Barresi, and S. H. Devoto, Devel-

$503 \quad$ opmental Dynamics 219, 287 (2000)

504 [28] D. Soroldoni, et al. Science 345, 222 (2014)

505 [29] F. J. van Eeden, et al. Development 123, 153 (1996).

506 [30] F. Cortes, et al. Developmental Cell 5, 865 (2003)

507 [31] A. K. Maurya, et al. PLoS Genetics 9 (2013).

508 [32] X. Wang, et al. Biology Open 2, 1203 (2013)

509 [33] A. Pagnon-Minot, et al. Developmental Biology 316, 21

$510 \quad(2008)$

511 34] Y.-Y. Lin, et al. Human Molecular Genetics 20, 1763

$512 \quad(2011)$

513 [35] J. Odenthal, et al. Development 123, 103 (1996).

514 [36] C. Anderson, et al. Molecular bioSystems 3, 51 (2007)

515 [37] S. H. Devoto, E. Melançon, J. S. Eisen, and M. Wester-

516 field, Development 122, 3371 (1996)

517 [38] C. Wolff, S. Roy, and P. W. Ingham, Current Biology 13, $518 \quad 1169$ (2003).

519 [39] M. Durande, S. Tlili, T. Homan, B. Guirao, F. Graner, and H. Delanoë-Ayari, arXiv:1810.11652 (2018)

521 [40] B. D. Crawford, C. A. Henry, T. A. Clason, A. L. Becker, and M. B. Hille, Molecular Biology of the Cell 14, 3065

$232003)$.

24 [41] D. Julich, A. P. Mould, E. Koper, and S. A. Holley, De- 
$525 \quad$ velopment 136, $2913(2009)$.

526 [42] K. Tawada and K. Sekimoto, Journal of Theoretical Bi-

$527 \quad$ ology 150, $193(1991)$

528 43] S. Walcott and S. X. Sun, Proceedings of the National

$529 \quad$ Academy of Sciences 107, 7757 (2010).

530 [44] M. Smutny, et al. Nature Cell Biology 19, 306 (2017).

531 [45] R. Farhadifar, J.-C. Röper, B. Aigouy, S. Eaton, and

${ }_{532}$ F. Jülicher, Current Biology 17, 2095 (2007)

533 [46] A. Mongera, et al. Nature 561, 401 (2018)

534 [47] J. Prost, F. Julicher, and J.-F. Joanny, Nature Physics $535 \quad \mathbf{1 1}, 111(2015)$

536 [48] F. Graner, B. Dollet, C. Raufaste, and P. Marmottant, ${ }_{537}$ European Physical Journal E 25, 349 (2008).

538 [49] S. Tlili, et al. Eur. Phys. J. E 38, 33/1 (2015)

539 [50] G. B. Blanchard, et al.,Nat. Methods 6, 458 (2009).

540 [51] S. E. Windner, N. C. Bird, S. E. Patterson, R. A. Doris, $541 \quad$ and S. H. Devoto, Biology Open 1, 806 (2012).

542 [52] K. McDole, et al. Cell 175, 859 (2018).

543 [53] A. Doostmohammadi, J. Ignés-Mullol, and J. M. Yeo544 mans, Nature Communications 9, 3246 (2018).

545 [54] K. Kawaguchi, R. Kageyama, and M. Sano, Nature 545,

$546 \quad 327$ (2017)

547 [55] G. Duclos et al., Nature Physics 14, 728 (2018).

548 [56] B. Bénazéraf, et al. Development 144, 4462 (2017).

549 [57] M. J. Barresi, H. L. Stickney, and S. H. Devoto, Devel$550 \quad$ opment 127, 2189 (2000).

551 [58] S. G. Sheela, W. C. Lee, W. W. Lin, and B. C. Chung,

552 Developmental Biology 286, 377 (2005).

553 [59] J. Malicki, et al. Development 123, 263 (1996) 
a

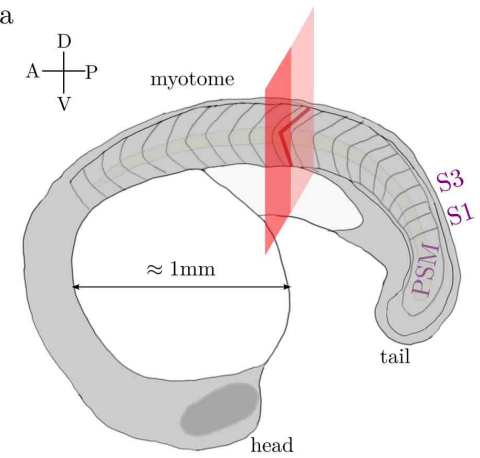

d
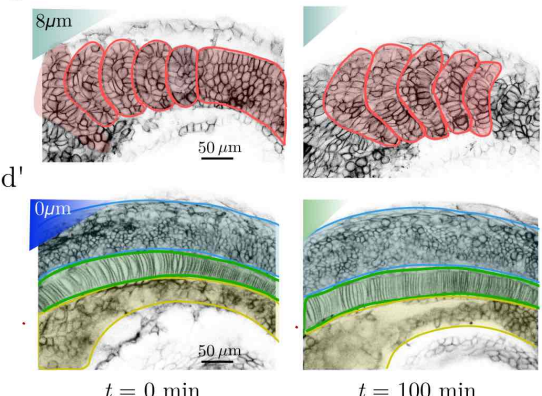

$t=100 \mathrm{~min}$ b

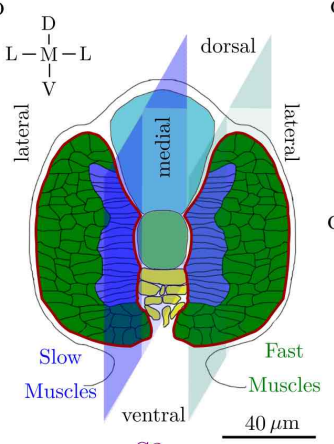

c

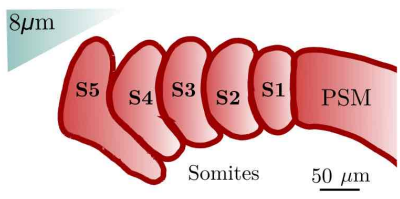

$c^{\prime}$

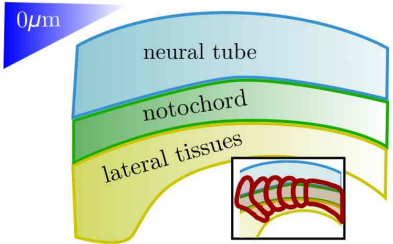

f
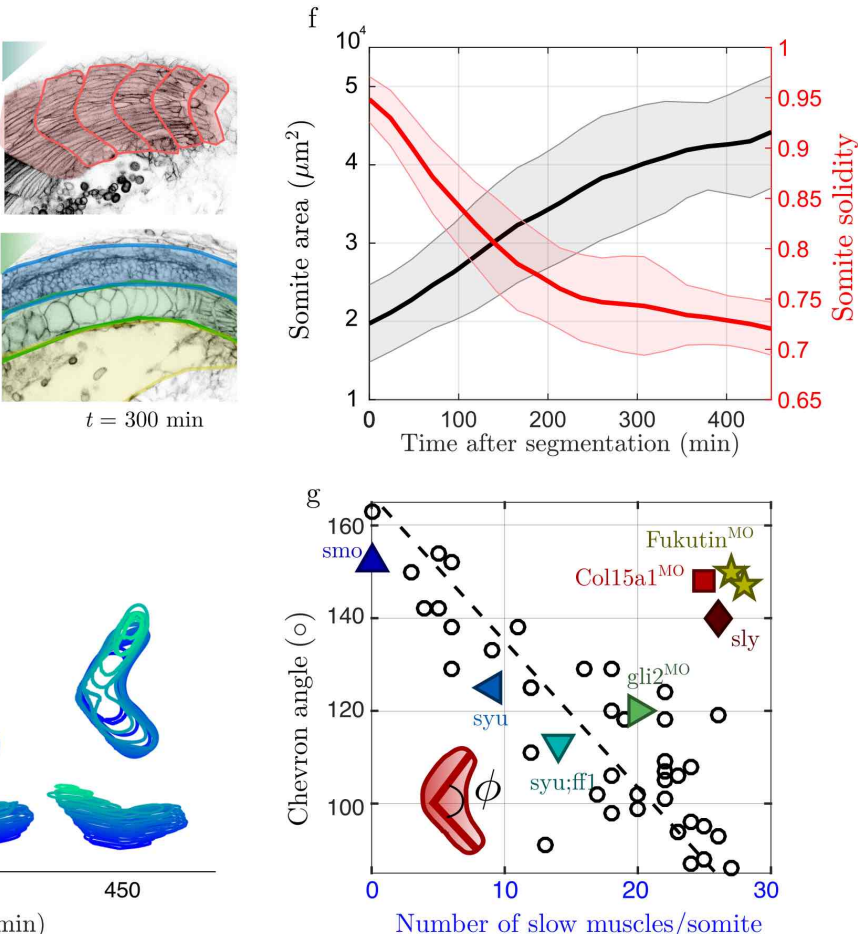

j

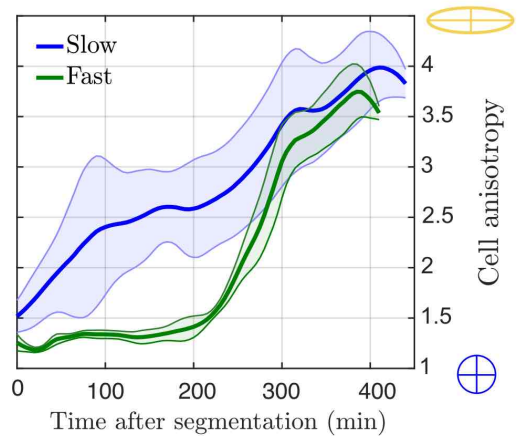

height from medial to lateral $(\mu \mathrm{m}) \boldsymbol{\omega}_{0}^{40}$

$A-\left.\right|_{V} ^{D} P$
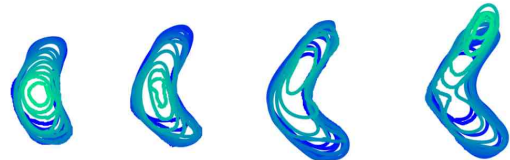

$\mathrm{A} \underset{\mathrm{M}}{\mathrm{L}} \mathrm{P}$

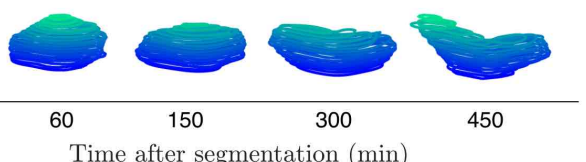

h

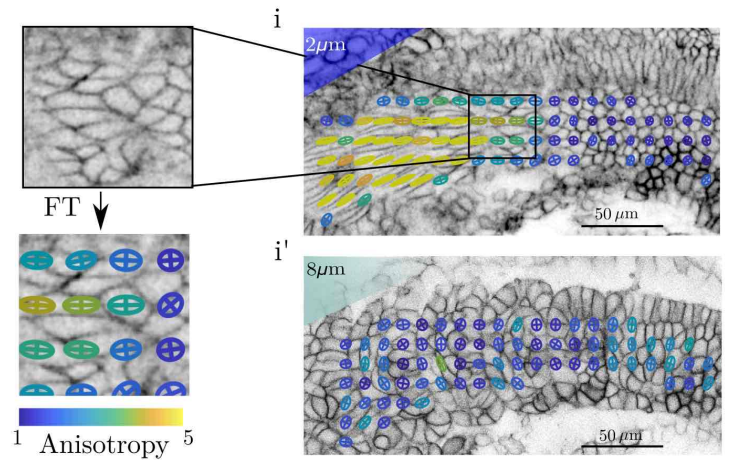


Figure 1. The chevron architecture of the myotome emerges early after segmentation from the PSM (a) Sketch of a 21-somite zebrafish embryo. Red plane: transverse plane to the anterior-posterior direction. Two somites, at stages S1 and S3, are highlighted. (b) Sketch of a S3-stage somite $(t \approx 90$ min post segmentation) in the transverse plane; dark blue (light green) cells are future slow (fast) muscle cells respectively. The dark and light blue planes represent the cross-sectional views shown in $\mathbf{c}$ and $\mathbf{d}$. The notochord is at the centre (grey circle), with the neural tube located more dorsally. Ventral tissues not shown for clarity. (c-c') Cartoon of somite shape in transverse view (c, plane lying $z=8 \mu \mathrm{m}$ from notochord) and underlying tissues (c', plane crossing the notochord, neural tube and ventral tissues. Inset shows shape of somites superimposed on underlying tissues. (d-d') Confocal images and superimposed contours of (d) somites and PSM (red lines) and (d') neural, notochord and mesoderm tissues (blue, green and yellow lines, respectively) at $t=0,100,300$ min post segmentation from PSM for the central somite shown in first panel. (e) 3D evolution of somite shape after segmentation from PSM of a representative wildtype embryo shows spreading of somite in DV-axis and emergence of chevron shape at $\sim 150 \mathrm{~min}$. (f) Cross-sectional area and solidity (i.e. the ratio of the somite area over the area of its convex hull) of segmented somites for the most medial layer of future fast muscle fibres (as in $\mathbf{d}$ ) as a function of time after segmentation. Shaded regions represents \pm 1 s.d.. (g) Chevron angle (in degrees) in the layer of most medial future fast muscle fibres against number of slow muscle cells per chevron. Black circles: Cyclopamine treated embryos at different concentrations. Triangles: morpholinos and mutants affecting cell differentiation (dark blue up $\triangle$ : smo [57, light blue left $\triangleleft$ : syu [58, cyan down $\nabla$ : syu+ff1 [58, and green $\triangleright$ : gli2 ${ }^{M O}$ [38]). Morpholinos or mutants altering tissue integrity (dark yellow star $\star$ : Fukutin [34], light red square $\square$ : Col15a1a ${ }^{M O}$ [33], dark red diamond $\diamond:$ sly [29, [59]). See Methods Table 1 for further details. (h) Fourier transform image analysis method provides a cell elongation field, with the anisotropy represented by ellipsoids (Methods). Cell elongation is along the major axis of the ellipse. (i) Elongation map of future slow (i) and fast (i') muscle fibres. (j) Mean cell anisotropy as a function of time post segmentation for future slow muscle fibres (i, blue) and for the layer of most medial future fast muscle fibres (i', green). Shaded regions represent \pm 1 s.d.. In (f) and (j): average is performed over 11 somites from 6 embryos. 
a

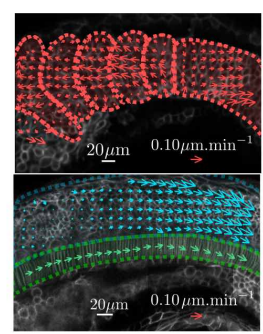

d

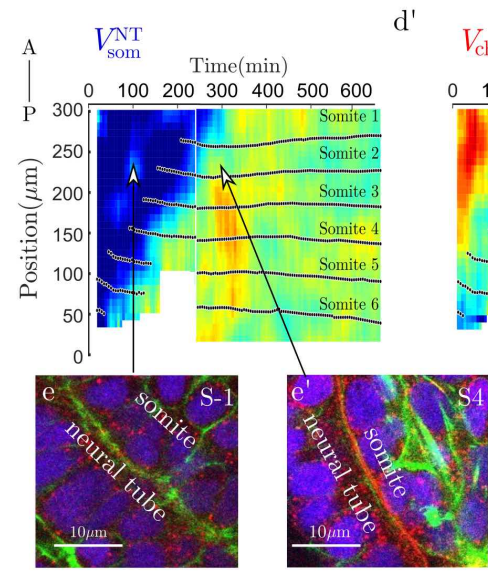

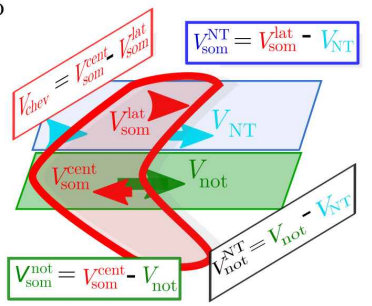
$V_{\text {chev }}$ Time $(\min )$ $100 \quad 200 \quad 300 \quad 400500600$ c

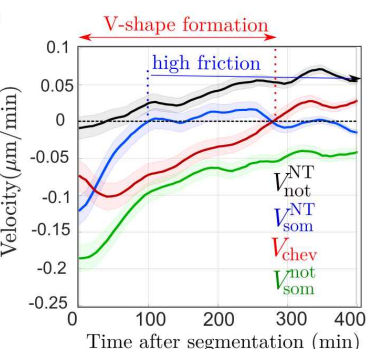

$d^{\prime \prime}$

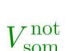
$V_{\mathrm{som}}^{\text {not }}$

Time $(\min )$

$0 \quad 100 \quad 200 \quad 300 \quad 400500600$

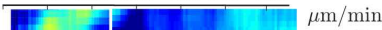

g

S 3-S 2
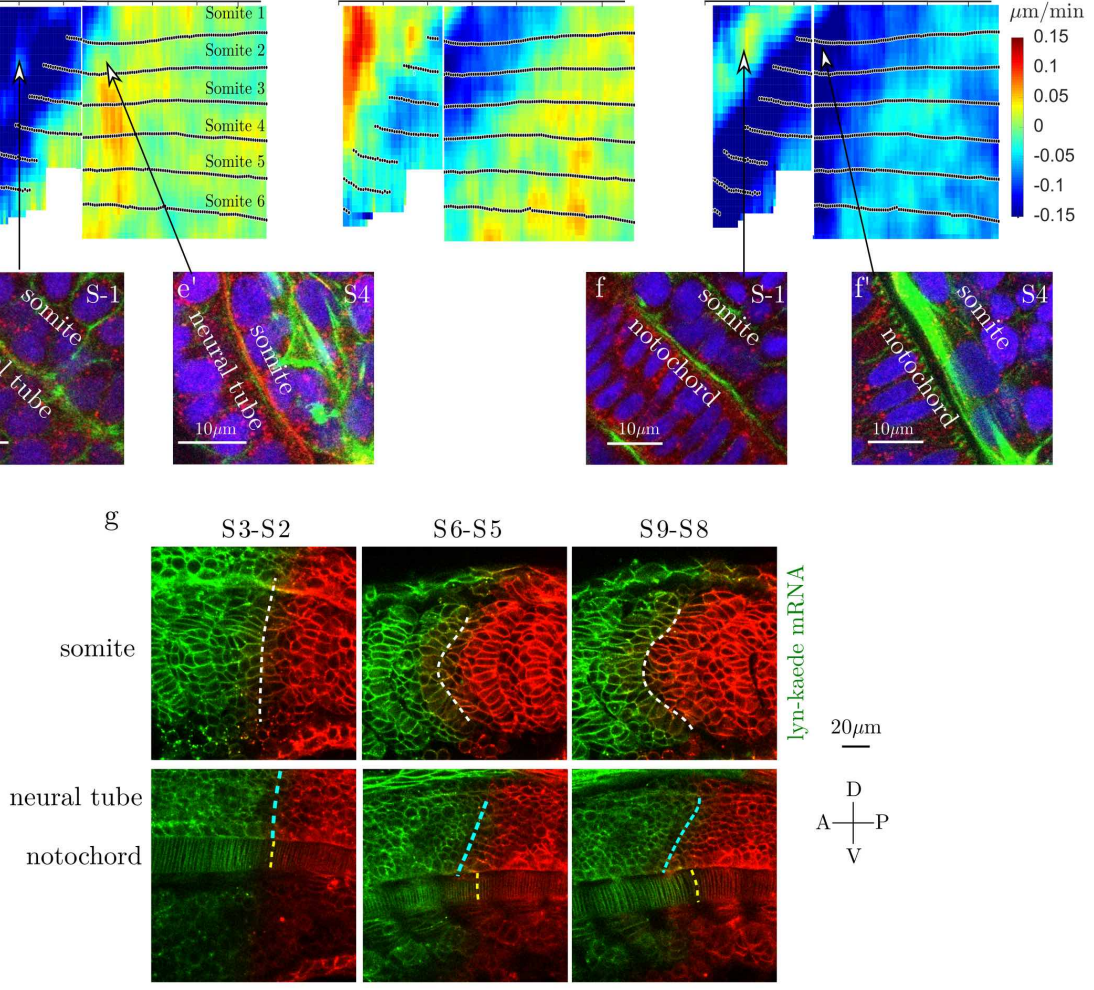

S9-S8

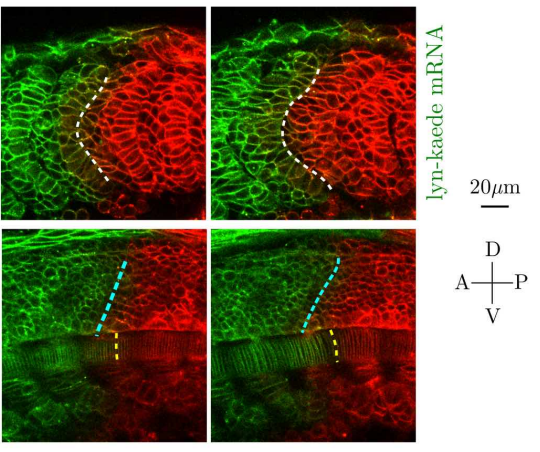

Figure 2. Differential tissue flow and heterogeneous mechanical coupling between tissues correlates with the emergence of a chevron shape. (a-a') Velocity fields estimated by Optic Flow (Methods) within the somite (red arrows, a), neural tube (cyan, $\mathbf{a}^{\prime}$ ) and notochord (green, b'). (b) Definition of the mean anterio-posterior (AP) velocities within each tissues: neural tube ( $N T$, cyan), notochord (not, green) and somites (som, red). (c) Evolution of the relative tissue AP velocities (average performed on $n=11$ somites from $N=5$ embryos) after segmentation from the PSM. Negative values of the shear strain rate $V_{\text {chev }}$ represent the period of chevron shape emergence. Near zero values of $V_{\text {som }}^{\mathrm{NT}}$ for $t>100$ min post-segmentation indicate the onset of a large friction between the notochord and somites. Shaded regions represent \pm 1 s.d. (d-d") Kymographs of shear velocities $V_{\mathrm{som}}^{\mathrm{NT}}, V_{\text {chev }}$ and $V_{\mathrm{som}}^{\mathrm{not}}$ shows somite-to somite reproducibility of the features identified in (c). Each panel from two embryos, with stitching at $t=220 \mathrm{~min}$. Black dots indicate the position of each somite centre of mass along the AP axis, with somite labelling representing somite number with respect to the start of the movie. In (d'), negative shear (blue coloured region) indicates the region where the chevron shape emerges in the somite. (e-f) Confocal images of actin (green), laminin (red) and nuclei (blue) in the transverse plane to the AP-axis for somites $S$-1 and $S 4$ (scale bar: $10 \mu \mathrm{m}$ ); (e) Closeup of the somite/neural tube interface. Arrows highlight correlation between actin and laminin localisation with the corresponding tissue-tissue flows shown in d. (f) Closeup of the somite/notochord interface. Arrows highlight correlation between actin and laminin localisation with the corresponding tissue-tissue flows shown in d". (g) Lyn-Kaede showing relative movement of the somites (top) with respect to the underlying notochord and neural tube (lower) from S2 to S9, with photo-switching of Kaede performed at S2 stage in the more posterior somites, Methods. Dashed lines highlight interfaces between the two fluorescent regions. 
bioRxiv preprint doi: https://doi.org/10.1101/505123; this version posted December 22,2018 . The copyright holder for this preprint (which was not certified by peer review) is the author/funder. All rights reserved. No reuse allowed without permission.

a

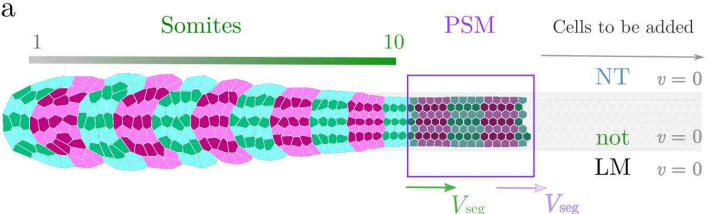

C

Spreading
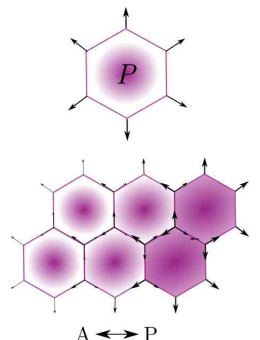

$c^{\prime}$

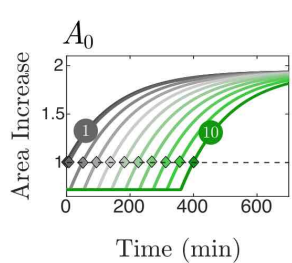

$c^{\prime \prime}$

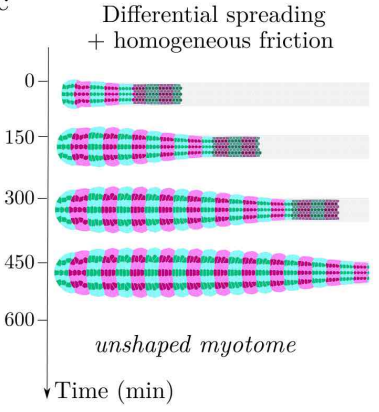

d

Differential friction

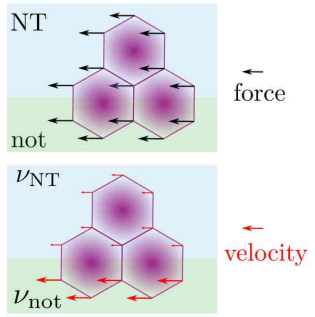

$\mathrm{d}^{\prime}$

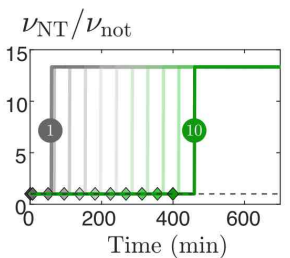

$d^{\prime \prime}$

+ differential friction

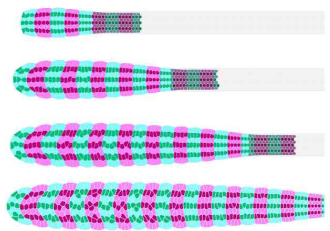

$U$-shaped myotome

stable, small angle $\mathrm{b}$

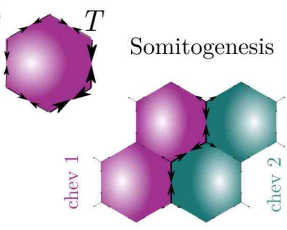

$\mathrm{e}$

Active stress
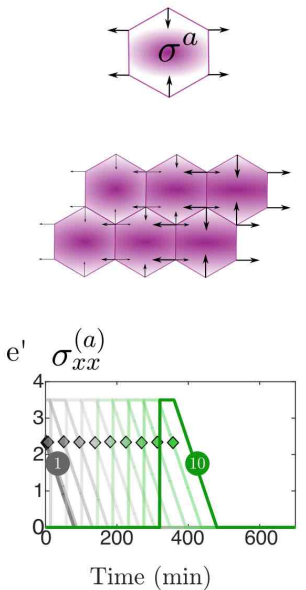

$e^{\prime 1}$

+ active stress

5
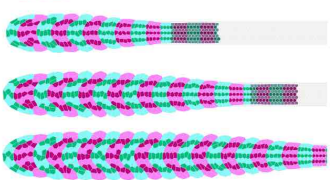

$V$-shaped myotome stable, large angle

f

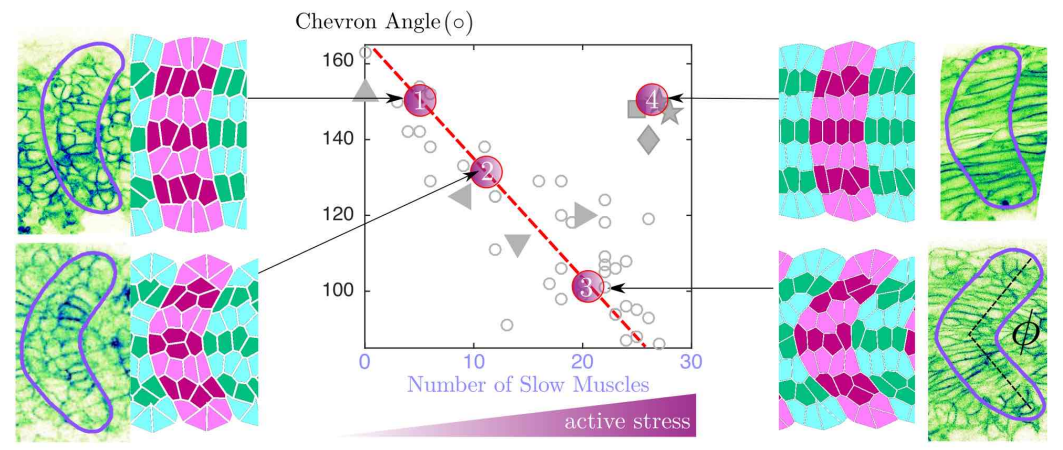


Figure 3. The chevron shape emerges in a vertex model incorporating somite spreading, differential friction and active stress. (a) Simulated geometry used in model. The number of simulated cells increases with time as new cells are progressively added from the tailbud region (highlighted by purple region); magenta (green) cells belong to somite number $2 N(2 N+1)$ respectively. New somites appear at a velocity $V_{\text {seg }}=1$ somite $/(30 \mathrm{~min})$. (b-e) Principle elements included within the vertex model: (b) Somite segmentation is implemented through an increased tension at the somite compartment boundaries. (c) Differential spreading is implemented through a wave of increased cellular pressure along the AP axis, leading to a spatial modulation of outward forces (black arrows). (c') Exponential increase in the somite target area as a function of time, based on experimental measurements. Grey curve (dark green) corresponds to first (last) somite formed in simulation (diamonds indicate timing of segmentation of specific somite from PSM, a). (c") Simulations with differential spreading only (i.e. homogeneous friction): somites do not buckle. (d) The vertex displacement (red arrow) is spatially modulated by an inhomogeneous friction coefficient $\nu$, where $\nu=\nu_{\mathrm{NT}}=\nu_{\mathrm{LM}}$ for vertices over the neural tube and ventral tissues; and $\nu=\nu_{\text {not }}$ otherwise. (d') The ratio of friction between the somite and neural tube and the somite and notochord, implemented as a step function (related to Fig. 3e-f). (d") Simulations with somite spreading and differential friction: somites fail to form a long-ranged sharp chevron shape. (e) An imposed bulk active stress $\sigma^{(a)}$ leads to elongation forces along the AP axis (black arrows). (e') Active stress is set to be maximal for each somite soon after segmentation, corresponding to slow muscle fibre elongation. (e") Simulations with active stress and differential friction (wildtype case): somites acquire a stable chevron shape. (f) Comparison of experimentally measured chevron angle (Fig. 1g) with the angle measured for four simulation outcomes. Only the active stress level is varied from points 1 to 3 (all other parameters fixed), describing embryos treated with $50 \mu$ mol (1), $10 \mu \mathrm{mol}$ (2) of Cyclopamine and wildtype embryos (3). (4) corresponds to the homogeneous friction case, describing the perturbed tissue-tissue coupling of Col15a1a ${ }^{M O}$. 
a

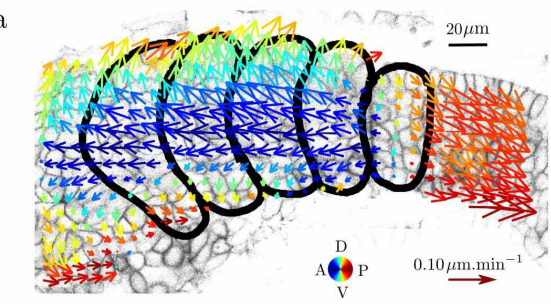

$\mathrm{b}$

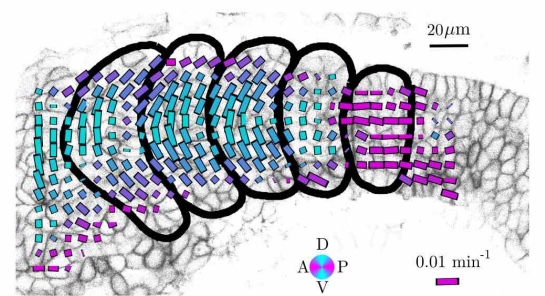

$\mathrm{a}^{\prime}$

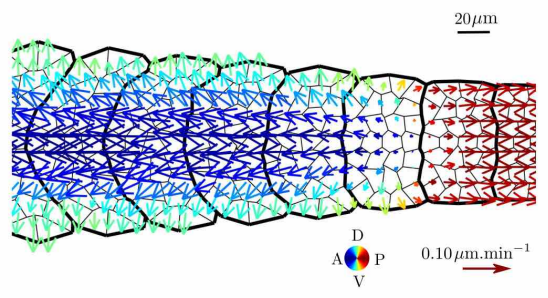

$b^{\prime}$

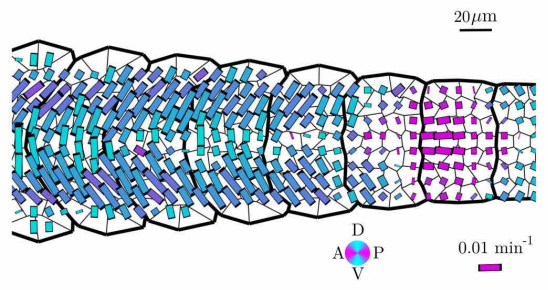

c

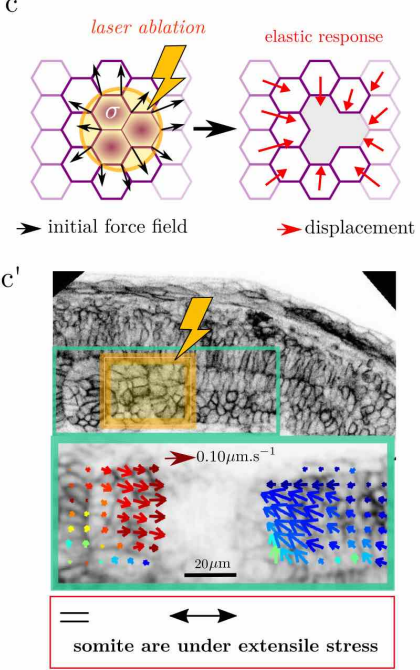

d

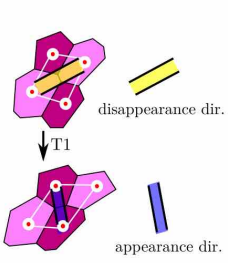

d' Somite A

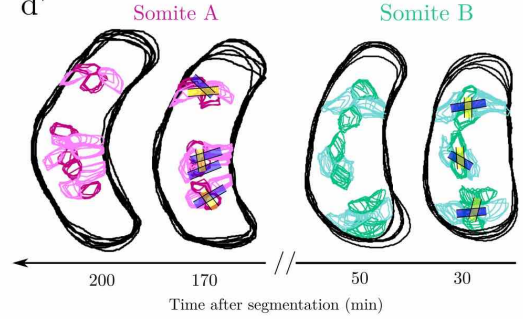

e

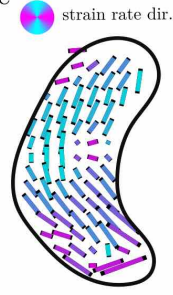

f

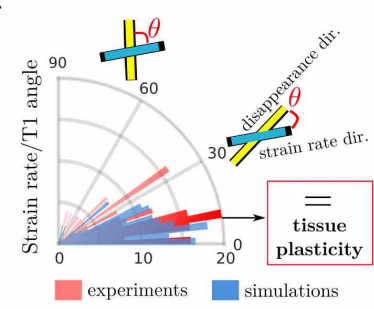

Figure 4. Model accurately predicts forces within the somite and orientation of cellular rearrangements. (aa') Comparison of velocity field in (a) experiments and (a') simulations, measured using optic flow, Methods. Arrow colour represents direction and length represents speed. (b-b') Comparison of the anisotropic component of the strain rates (ASR) in (b) experiments and (b') simulations (magenta: AP orientation; cyan: DV orientation). Bar color represents orientation and length represents the strain rate magnitude (see Methods). (c) Cartoon of predicted relaxation direction upon ablation of somitic tissue. (c') (Top) Laser ablation (yellow box) of somites at stage S0 and S1. (Bottom) Zoomed in region highlighted above, with arrows representing tissue velocity from optic flow analysis in the $10 \mathrm{~s}$ after ablation. Colour coding as a). (d) Scheme of cellular rearrangements, with cells losing contact joined by yellow bar and cells forming new contacts by blue bar. (d') Experimental examples of 3D cellular rearrangements at different somite stages for 2 somites. (e) Time and ensemble averaged ASR ( $n=4$ somites). (e') Accumulated cell rearrangements orientations (across 4 somites) superimposed on the ASR map. (f) Rose plot alignment of cellular rearrangement with ASR in experiments ( $n=44$ from 4 somites) and simulations ( $n=60$ from 6 simulated somites). 